incorporated in the software provided with the microcomputer, which can facilitate office work and repetitive administrative tasks, such as letters of appointment and addressing envelopes.

The beauty of local microcomputer based case registers is that they can all talk to a centralised database maintained on a more powerful computer. In this way a number of geographically disparate sites can run their own local systems, customised to their own operational needs and interests, whilst maintaining a common core of clinical information that can be pooled for a more comprehensive analysis. This kind of link-up can be achieved cheaply over the telephone line with the purchase of a modem.
In the fairly near future, with the growing availability of multi-user operating systems for microcomputers (e.g. Xenix, Unix) another generation of database management software will become available to local case register projects. Here it will be possible not only to operate highly sophisticated local case registers, with theoretically unlimited storage capacity and complex query options, but also to connect up a number of local sites 'on-line'. Each site could then share a common database where the records relating to each service user are readily available within seconds. We would hope that this type of development would generate the same kinds of 'spin-off' benefits we enjoyed at 608.

\title{
Ethnic Minorities and the Mental Health Act Patterns of Explanation
}

\author{
Roland Littlewood, Senior Lecturer, University Department of Psychiatry, All Saints Hospital, Birmingham
}

Explanations of the over-representation of black psychiatric patients under the Mental Health Act tend to emphasise either the particular psychopathology of black patients or alternatively to locate it within the decisions of individual psychiatrists and police in a racist society. Both contain specific (conspiratorial) and general (cultural) variants. The two models are incomplete in themselves.

Investigations of British hospital records have demonstrated a disproportionate number of black patients, both born in Britain and in the Caribbean and West Africa, who are involuntary patients in psychiatric hospitals under the Mental Health Act. Some studies have found that Asianborn patients are also over-represented. The degree of overrepresentation varies but most studies suggest that it is between two and three times the white UK-born rate; whereas about $8 \%$ of white UK-born patients in psychiatric hospitals and units are involuntary, the figure for black patients is about 20 to $30 \%$. As far as we know this pattern is similar in different areas of Britain. To an extent the figures may be explained by increased rates of schizophrenia in the black community relative to other diagnoses; this is likely to be associated with involuntary admission. There is evidence however that the increase is independent of diagnosis, whilst the frequency of the diagnosis of schizophrenia in minorities has been called into question.

Research in this area tends to be orientated towards the simple collection of data in ignorance of the implicit models which govern its collection. In this paper I shall consider the two broad groups of explanations which have been offered: one implicates factors in the patients themselves and in black society, the other is concerned with the practice of psychiatry in the context of black/white relations.

Explanations orientated to the black patient

Not surprisingly these are the explanations offered by psychiatrists.
A. The specific suggestion that psychiatric illness in black patients is different. Among the terms which are commonly used are 'florid', 'acute' and so on; black patients are regarded as more antisocial and dangerous and thus legitimately more likely to be placed on sections of the Mental Health Act, whilst the police are more often involved in the admission of black patients in general. Although there are few studies on the numbers of black patients in locked wards, secure units and special hospitals (partly because of the reluctance of the Home Office and individual psychiatrists to be accused of racism), it appears that black patients are over-represented in these settings too. Why should the presentation of psychiatric illness in blacks be more anti-social than in whites? Amongst the arguments put forward (usually by analogy with suggestions made by colonial psychiatrists in Africa and elsewhere) are that:

(i) The illness is precipitated by organic factors such as anaemia and therefore the symptoms are more 'toxic' and delirious and there is less responsiveness to social context. There is no evidence that this is the case, although the nutritional status of minority psychiatric patients in Britain has not been investigated, perhaps surprisingly given the medical preoccupation with the diets of Rastafarians and Hindus.

(ii) Different cultures do have different patterns of mental illness and another explanation has been that the illness in the black British community (which is regarded as a separate culture) represents a 'migration' of the illness itself. (The majority of patients in Caribbean psychiatric hospitals are involuntary). This offers a sort of acculturation model: black illnesses will come to resemble indigenous white ones; or an evolutionary one-with industrialisation, psychopathology becomes more 'psychologised', private and internalised. A variant of this thesis is that illnesses are somehow shaped by the ambient culture and, as the Caribbean is regarded in general as akin to West Kingston, 
the illness itself is more 'aggressive' and antisocial. This of course resembles similar models derived in the areas of law and education to explain prison statistics and examination results: in other words problems are seen to lie in the minority culture itself which is regarded as pathogenic if not pathological. However, black patients of various ethnic groups (including South Asians) are over-represented on Mental Health Act Sections, some of whom are underrepresented in other measures of what may be termed 'social deviance'; nor are the various societies of the Indian sub-continent described by British professionals as 'antisocial' in the way the Caribbean is perceived. In a small study of the offences which led courts to refer patients to psychiatric hospitals, black patients were no more likely to have committed violent offences against others than were whites. However transfer of patients to secure units is related to perceived 'unco-operativeness' and is a more common experience for black patients.

(iii) What may be termed a 'liberal' variant of the same type of explanation locates the aggressive proclivity in the communities' response to racism in British societies. Similar patterns of compulsory treatment are found in other disadvantaged minority groups elsewhere in the world, independent of their own culture. In favour of this argument is the fact that the children of non-white migrants are as likely as their parents to be involuntary patients. Patterns of psychopathology in migrants in any case quickly come to resemble the prevalent local patterns; teenage immigrants to Britain from rural South Asia may take an overdose after a few years residence here.

(iv) The older type of explanation, which implicated inherited 'racial' characteristics as significant, has not been suggested by anyone in the current debate. There is in fact no evidence that any broad patterns of cross-cultural differences in mental illness can be ascribed to population genetics.

B. Community attitudes. Another more general explanation offered by psychiatrists of the figures is that the differences can be attributed less to the individual patient than to the minority communities' perception and conceptualisation of psychopathology. According to this model mental illness is particularly stigmatised in minority communities, in part because of pre-migration folk models of mental illness, and in part because in the pre-migration culture there are anyway fewer and poorer psychiatric facilities which are reserved for the more severely ill and the antisocial. The presumed consequences are that:

(i) The potential patient is less willing to come into a mental hospital.

(ii) The patient's own model of mental illness is more antisocial.

(iii) Recourse to psychiatric facilities occurs later in the course of the illness when the patients are less likely to regard themselves as ill and thus accept treatment.

(iv) Whilst it may be expected that the experience of being black in Britain is likely to lead to an increase in mental illness, the total rates of hospital admission are broadly similar in most groups, suggesting that perhaps only the most severely ill black patients are actually admitted. These are likely to be disproportionately 'antisocial'.

In favour of the 'stigmatisation' argument is that black psychiatric patients appear to have a poorer relationship with doctors and to be more likely to miss appointments. However white migrants from countries with the same type of psychiatric facilities as those found in India or the Caribbean are no more likely to be compulsorily detained than the white British-born. More significantly, although there are assumptions among psychiatrists of the stigmatisation of the mentally ill by minority groups, there is little evidence for this with the exception of a short and relatively inconclusive study in Birmingham. Indeed we know little about popular conceptualisations of mental illness in any group including the white British. There is however evidence that the prognosis of serious mental illness is actually better outside Europe; recent studies in rural India suggest that schizophrenic patients are less excluded and invalidated by their families than in Britain. As to the prognosis of serious mental illness in different ethnic groups inside Britain, we again have no information. A more significant objection to this argument is that explanations based on premigration culture are infinitely elastic and of dubious validity to the situation here and now: over-representation under the Mental Health Act is as common in the Britishborn black community as it is in their immigrant parents.

\section{Explanations which emphasise the role of the poychiatrist}

A different set of explanations emphasises the political role of psychiatry or expresses the immediate subjective experience of being a black psychiatric patient in Britain. Many of the explanations here are strikingly similar to those suggested by professional psychiatry, with the distinction that the arrow of causality is reversed; thus black psychiatric outpatients may indeed miss more appointments but this is perhaps because they receive an inferior service as in other areas of medicine.

(i) A common suggestion is that the police are overtly racist and selectively pick out non-mentally ill black people in the streets, and take them to a psychiatric hospital under Section 136 of the Mental Health Act as an alternative to arrest. This certainly does occur from my own clinical experience in East London and is commonly mentioned by the black community.

(ii) Black patients, however, are over-represented on all types of Mental Health Act Sections: not only from the police but those signed by general practitioners, psychiatrists and courts, and this over-representation is of a similar order for all Sections. This would fit in with the suggestion that, in a racist society, it is psychiatry which has a specific role as the social moderator of conformity, patrolling the uneasy borderline between illness and political dissent. This is supported by the common assertion, not statistically substantiated but which is probably true, that members of Ras Tafari are particularly likely to be psychiatric patients; a variety of studies show that, with the possible exception of Jehovah's Witnesses, membership of 
religious groups is usually associated with better mental health.

(iii) A more 'liberal' variant of this theory is that psychiatrists are just basically incompetent in diagnosis and this is supported by the increased frequency of a change of diagnosis in patients from ethnic minorities. It appears likely that depression in the black community may be diagnosed as schizophrenia and that patients are thus more likely to be compulsorily detained because of the perception of schizophrenia as inherently more antisocial.

(iv) Psychiatrists themselves would deny that they consciously and selectively penalise black patients whilst agreeing that they are dealing with the casualties of disadvantage (and at times offering in the press crude stereotypes of minority patients). Wherever such practice is located it is not likely to be in conscious overt racism. Whether this is also true of the police may be doubted. In the only study which looked at the ethnicity of psychiatrists, admittedly one of limited scope, this did not appear to be associated with the treatment of patients of different ethnic groups. Although the majority of junior psychiatrists are themselves non-white this does not seem to be related to the overall findings, suggesting again that racialism does not operate at an overt, conscious level. There is however ample evidence that within the NHS itself black workers are underprivileged.

(v) No-one has attempted to deal with any degree of sophistication with how covert (institutional) racialism operates in psychiatry. Is psychiatry "a crucial ... new operational method", a specific form of indirect control in a racist society, or is its use of the Mental Health Act merely part of the same package of oppression and disadvantage as unemployment and poor housing, such that to be an involuntary patient is merely on a par with the relative lack of access to psychotherapy or counselling, and with higher dosages of medication? Against this 'general racialism argument' is the specific historical role of psychological and psychiatric theories in justifying slavery, colonialism and racism. Supporting the argument that psychiatry merely reflects or represents general non-specific racialism are the other indices of medical disadvantage, such as infant mortality and infectious diseases associated with malnutrition, which demonstrate an over-representation of non-white groups. Also in favour is the fact that the 'medical' Sections 2,3,4 of the Mental Health Act have to be signed not only by doctors but also by members of the patient's family or by social workers. If a differential use of the Mental Health Act is located in conscious discriminatory practices, one might expect that black families would be reluctant to consign their relatives to mental hospitals and that more of the Sections for black patients would be signed by social workers than Sections for white patients. So far as we know this is not the case: a quick look at $\mathbf{3 4}$ completed forms in Birmingham suggests that there is no difference. Families can be pressurised of course into signing Sections by the professionals; however in the majority of cases when police have been called to a black person for psychiatric reasons, it has been at the request of neighbours or the family themselves.

\section{A Critical Theory?}

The idea that racism in Britain operates solely in terms of deliberate unequal treatment might appear rather naive. Racialism is likely to be mediated by implicit assumptions of the type I have outlined earlier, part of a shared everyday view of the world, both on the part of those who benefit from the system but also of those who do not: thus the attitudes to 'cannabis psychosis' among the black British community as a whole and among white psychiatrists appear identical; in many cases they appear to be prejudiced and unwarranted by clinical findings.

Both types of explanation appear inadequate in themselves: the psychiatrist's perception of violence in the patient and the perception of the psychiatrist as an agent of racism. Both are conspiratorial theories which locate interaction between groups in society in one element of it alone. Whilst that might seem a rather obvious conclusion, the explanations considered do tend to fall discretely into one of these two groups. On certain points, simple empirical data are certainly required, but such data need to be informed by a more developed theory. Rather than convergent data (comparing groups or independent sets of patients and locating the differences in these groups), we need divergent data in which we can perceive how different groups interact. Data, like the expression of mental illness itself, are not independent of constraining theories but part of a total social context which includes such theory. An analysis of psychiatry's functions of social control in the black community necessitates a consideration of its relations with other welfare agencies, courts, probation services, prisons and immigration control procedures; it is unlikely to be independent of policies and practices in such areas as social work or fostering.

Objections to the first approach are perhaps the most serious in that it may be reasonably questioned whether psychiatric theory and practice which themselves represent part of the interaction can provide an adequate tool for describing it. The second perspective too, particularly in its simplest conspiratorial form, is also partial, nor is there any reason to suppose that the immediate experiences of racism or the individual responses to it themselves comprise any type of privileged theory. What remains to be demonstrated is the nature of the social transaction whereby psychiatrists perceive their black patients as more dangerous. We have yet to develop a critical theory of the type so fruitful in women's studies, one which is independent both of psychological theories and personal experience, but which shows how both have originated historically and how they constitute each other.

A full bibliography for this paper is available from the author. 